\title{
Facility Location and the Geometric Minimum-Diameter Spanning Tree
}

\author{
Joachim Gudmundsson \\ Herman J. Haverkort \\ Sang-Min Park \\ Chan-Su Shin \\ Alexander Wolff
}

institute of information and computing sciences, utrecht university technical report UU-CS-2003-006 www.cs.uu.nl 


\begin{abstract}
Let $P$ be a set of $n$ points in the plane. The geometric minimum-diameter spanning tree (MDST) of $P$ is a tree that spans $P$ and minimizes the Euclidian length of the longest path. It is known that there is always a mono- or a dipolar MDST, i.e. a MDST with one or two nodes of degree greater 1 , respectively. The more difficult dipolar case can so far only be solved in slightly subcubic time.

This paper has two aims. First, we present a solution to a new data structure for facility location, the minimum-sum dipolar spanning tree (MSST), that mediates between the minimum-diameter dipolar spanning tree and the discrete two-center problem $(2 \mathrm{CP})$ in the following sense: find two centers $p$ and $q$ in $P$ that minimize the sum of their distance plus the distance of any other point (client) to the closer center. This is of interest if the two centers do not only serve their customers (as in the case of the $2 \mathrm{CP}$ ), but frequently have to exchange goods or personnel between themselves. We show that this problem can be solved in $O\left(n^{2} \log n\right)$ time and that it yields a factor-4/3 approximation of the MDST.

Second, we give two fast approximation schemes for the MDST, i.e. factor- $(1+\varepsilon)$ approximation algorithms. One uses a grid and takes $O^{*}\left(E^{6-1 / 3}+n\right)$ time, where $E=$ $1 / \varepsilon$ and the $O^{*}$-notation hides terms of type $O\left(\log ^{O(1)} E\right)$. The other uses the wellseparated pair decomposition and takes $O\left(n E^{3}+E n \log n\right)$ time. A combination of the two approaches runs in $O^{*}\left(E^{5}+n\right)$ time. Both schemes can also be applied to MSST and $2 \mathrm{CP}$.
\end{abstract}

\title{
1 Introduction
}

The MDST can be seen as a network without cycles that minimizes the maximum travel time between any two sites connected by the network. This is of importance e.g. in communication systems where the maximum delay in delivering a message is to be minimized. Ho et al. showed that there always is a mono- or a dipolar MDST [HLCW91]. For a different proof, see [HT95]. Ho et al. also gave an $O(n \log n)$-time algorithm for the monopolar and an $O\left(n^{3}\right)$ time algorithm for the dipolar case [HLCW91]. In addition, they showed that the problem becomes considerably easier when allowing Steiner points, i.e. to find a spanning tree with minimum diameter over all point sets $P^{\prime}$ that contain the input point set $P$. The reason is that there always is a minimum-diameter Steiner tree that is monopolar and whose pole is the center of the smallest enclosing circle of $P$. Thus the minimum-diameter Steiner tree can be determined in linear time [HLCW91].

The cubic time bound for the dipolar case was recently improved by Chan [Cha02] to $\tilde{O}\left(n^{3-c_{d}}\right)$, where $c_{d}=1 /((d+1)(\lceil d / 2\rceil+1))$ is a constant that depends on the dimension $d$ of the point set and the $\tilde{O}$-notation hides factors that are $o\left(n^{\varepsilon}\right)$ for any fixed $\varepsilon>0$. In the planar case $c_{d}=1 / 6$. Chan speeds up the exhaustive-search algorithm of Ho et al. by using new semi-dynamic data structures. Note however that $c_{d}$ tends to 0 with increasing $d$, while the asymptotic running time of the algorithm of Ho et al. does not depend on the dimension.

Note that in the dipolar case the objective is to find the two poles $x, y \in P$ of the tree such that the function $r_{x}+|x y|+r_{y}$ is minimized, where $|x y|$ is the Euclidean distance of $x$ and $y$, and $r_{x}$ and $r_{y}$ are the radii of two disks centered at $x$ and $y$ whose union covers $P$. On the other hand the discrete $k$-center problem is to determine $k$ points in $P$ such that the union of $k$ congruent disks centered at the $k$ points covers $P$ and the radius of the disks is minimized. This is a typical facility location problem: there are $n$ supermarkets and in $k$ of them a regional director must be placed such that the maximum director-supermarket distance is minimized. This problem is NP-hard provided that $k$ is part of the input [GJ79]. 
Thus, the main research on this problem has focused on small $k$, especially on $k=1,2$. For $k=1$, the problem can be solved in $O(n \log n)$ time using the farthest-point Voronoi diagram of $P$. For $k=2$, the problem becomes considerably harder. Using the notation from above, the discrete two-center problem consists of finding two centers $x, y \in P$ such that the function $\max \left\{r_{x}, r_{y}\right\}$ is minimized. Agarwal et al. [ASW98] gave the first subquadratic-time algorithm for this problem. It runs in $O\left(n^{4 / 3} \log ^{5} n\right)$ time.

In this paper we are interested in (a) a new facility location problem that mediates between the minimum-diameter dipolar spanning tree (MDdST) and the two-center problem and (b) fast approximations of the computationally expensive MDdST. As for our first aim we observe the following. Whereas the MDdST minimizes $|x y|+\left(r_{x}+r_{y}\right)$, the discrete two-center problem is to minimize $\max \left\{r_{x}, r_{y}\right\}$, which means that the distance between the two centers is not considered at all. If, however, the two centers need to communicate with each other for cooperation, then their distance should be considered as well - not only the radius of the two disks. Therefore our aim is to find two centers $x$ and $y$ that minimize $|x y|+\max \left\{r_{x}, r_{y}\right\}$, which is a compromise between the two previous objective functions. We will refer to this problem as the discrete minimum-sum two-center problem and call the resulting graph the minimum-sum dipolar spanning tree (MSST). As it turns out, our algorithm for the MSST also constitutes a compromise, namely in terms of runtime between the subcubic-time MDdST-algorithm and the superlinear-time 2CP-algorithm. More specifically, in Section 2 we will describe an algorithm that solves the discrete minimum-sum two-center problem in the plane in $O\left(n^{2} \log n\right)$ time using $O\left(n^{2}\right)$ space. For dimension $d<5$ a variant of our algorithm is faster than the more general $\tilde{O}\left(n^{3-c_{d}}\right)$-time MDST-algorithm of Chan [Cha02] that can easily be modified to compute the MSST instead.

In Section 3 we turn to our second aim, approximations for the MDST. To our knowledge nothing has been published on that field so far. ${ }^{1}$ We combine a slight modification of the MSST with the minimum-diameter monopolar spanning tree (MDmST). We identify two parameters that depend on the MDdST and help to express a very tight estimation of how well the two trees approximate it. It turns out that at least one of them is a factor- $4 / 3$ approximation of the MDST.

Finally, in Section 4 we show that there are even approximating schemes for the MDdST. More precisely, given a set $P$ of $n$ points and some $\varepsilon>0$ we show how to compute a dipolar tree whose diameter is at most $(1+\varepsilon)$ times as long as the diameter of a MDdST. Our first approximation scheme uses a grid of $O(E) \times O(E)$ square cells (where $E=1 / \varepsilon$ ) and runs Chan's exact algorithm [Cha02] on one representative point per cell. The same idea has been used before [BHP99, Cha00] to approximate the diameter of a point set, i.e. the longest distance between any pair of the given points. Our scheme takes $O^{*}\left(E^{6}+n\right)$ time, where the $O^{*}$-notation hides terms of type $O\left(\log ^{O(1)} E\right)$.

Our second approximation scheme is based on the well-separated pair decomposition [CK95] of $P$ and takes $O\left(E^{3} n+E n \log n\right)$ time. Well-separated pair decompositions make it possible to consider only a linear number of pairs of points on the search for the two poles of an approximate MDdST. If we run our second scheme on the $O\left(E^{2}\right)$ representative points in the grid mentioned above, we get a new scheme with a running time of $O^{*}\left(E^{5}+n\right)$. Both schemes can also be adjusted to approximate the MSST and the $2 \mathrm{CP}$ in linear time.

We will refer to the diameter $d_{P}$ of the MDST of $P$ as the tree diameter of $P$. We assume

\footnotetext{
${ }^{1}$ Very recently we were informed of $\left[\mathrm{SKB}^{+} 02\right]$ where the authors give an approximation scheme for the MDST that runs in $O\left(\varepsilon^{-3}+n\right)$ time using $O(n)$ space.
} 
that $P$ contains at least four points.

\section{The Minimum-Sum Dipolar Spanning Tree}

It is simple to give an $O\left(n^{3}\right)$-time algorithm for computing the MSST. Just go through all $O\left(n^{2}\right)$ pairs $\{p, q\}$ of input points and compute in linear time the point $m_{p q}$ whose distance to the current pair is maximum. In order to give a faster algorithm for computing the MSST, we need a few definitions. Let $h_{p q}$ be the open halfplane that contains $p$ and is delimited by the perpendicular bisector $b_{p q}$ of $p$ and $q$. Note that $h_{p q}, h_{q p}$, and $b_{p q}$ partition the plane. Let $\mathcal{T}_{p q}$ be the tree with dipole $\{p, q\}$ where all other points are connected to the closer pole. (Points on $b_{p q}$ can be connected to either $p$ or q.) Clearly the tree $\mathcal{T}_{p q}$ that minimizes $|p q|+\min \left\{\left|p m_{p q}\right|,\left|q m_{p q}\right|\right\}$ is a MSST.

The first important idea of our algorithm is to split the problem of computing all points of type $m_{p q}$ into two halves. Instead of computing the point $m_{p q}$ farthest from the pair $\{p, q\}$, we compute for each ordered pair $(p, q)$ the point $f_{p q} \in P \backslash h_{q p}$ that is farthest from $p$ ignoring the halfplane of $q$, see Figure 1 . We call $f_{p q}$ the $q$-farthest point from $p$. Now we want to find the tree $\mathcal{T}_{p q}$ that minimizes $|p q|+\max \left\{\left|p f_{p q}\right|,\left|q f_{q p}\right|\right\}$. This strategy enables us to reuse information.

Our algorithm consists of two phases. In phase I we go through all points $p$ in $P$. The central (and time-critical) part of our algorithm is the computation of $f_{p q}$ for all $q \in P \backslash\{p\}$. In phase II we then use the above form of our target function to determine the MSST.

The second important observation that helped us to reduce the running time of the central part of our algorithm is the following. Let $p$ be fixed. Instead of going through all $q \in P \backslash\{p\}$ and computing $f_{p q}$ we characterize all $q$ for which the $q$-farthest point $f_{p q}$ of $p$ is identical:

Lemma 2.1 If $x \in P$ is the farthest point from $p \in P$, then $x$ is the q-farthest point from $p$ if and only if $q \notin D(x, p)$, where $D(a, b)$ (for points $a \neq b$ ) is the open disk that is centered at $a$ and whose boundary contains $b$.

Proof: Since $x$ is farthest from $p, x$ is $q$-farthest from $p$ if and only if $x \in h_{p q}$. This is the case iff the angle $\alpha=\angle p c x$ in the midpoint $c$ of $p q$ is at most 90 degrees, see Figure 2. Due to the Theorem of Thales this is equivalent to $c \notin D(m, p)$, where $m$ is the midpoint of $p x$. Finally this is equivalent to $q \notin D(x, p)$, since $d(p, q)=2 d(p, c)$ and $D(x, p)$ is the result of scaling $D(m, p)$ relative to $p$ by a factor of 2 .

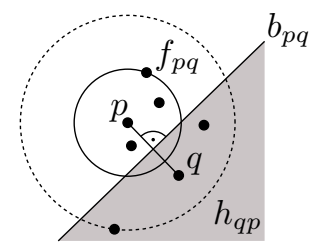

Figure 1: The $q$-farthest point $f_{p q}$ from $p$ is farthest from $p$ among all points closer to $p$ than to $q$.

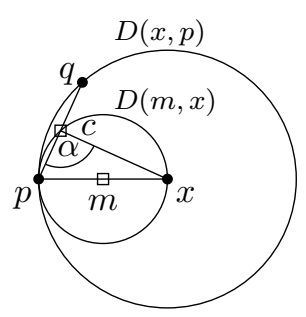

Figure 2: If $x$ is farthest from $p$ then $x$ is $q$-farthest from $p$ iff $q \notin D(x, p)$.

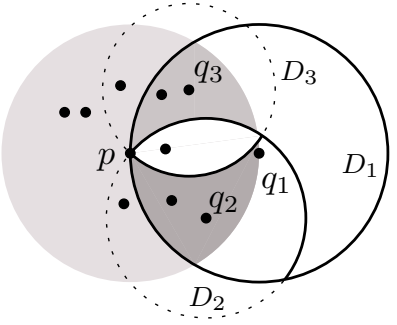

Figure 3: Labeling points with their $q$ farthest point. 
Using the above characterization we can label all points $q \in P \backslash\{p\}$ with the $q$-farthest point $f_{p q}$ as follows. We first sort $P$ in order of "decreasing" (i.e. non-increasing) distance from $p$. Let $q_{1}, q_{2}, \ldots, q_{n}=p$ be the resulting order. Label all points in the complement of $D\left(q_{1}, p\right)$ with $q_{1}$. Then label all unlabeled points in the complement of $D\left(q_{2}, p\right)$ with $q_{2}$. Continue this process until all points are labeled. Figure 3 visualizes the first three steps of this process. In that figure the areas shaded light, medium, and dark correspond to the areas in which all points are labeled with $q_{1}, q_{2}$, and $q_{3}$, respectively.

It remains to show how all points $q \in P \backslash\{p\}$ can be labeled with $f_{p q}$ efficiently. One approach would be to use dynamic circular range searching, which is equivalent to halfspace range searching in $\mathbb{R}^{3}$ [AM95]. The necessary data structure can be build in $O\left(n^{1+\varepsilon}\right)$ time and space. After each query with a disk $D\left(q_{i}, p\right)$ all points that are not returned must be deleted. The total time for querying and updating is also $O\left(n^{1+\varepsilon}\right)$. This would yield an $O\left(n^{2+\varepsilon}\right)$-time algorithm. We will show that we can do better in the plane. However, it is not clear how our results can be generalized to higher dimensions. For dimensions $d \in\{3,4\}$ computing the MSST with range searching takes $O\left(n^{2.5+\varepsilon}\right)$ time [AM95] and thus is still faster than Chan's $\tilde{O}\left(n^{3-c_{d}}\right)$-time algorithm [Cha02], where $c_{d}=1 /((d+1)(\lceil d / 2\rceil+1))$.

Lemma 2.2 Given a set $P$ of $n$ points in the plane and given $n$ disks $D_{1}, \ldots, D_{n}$ that all touch a point $p$, there is a data structure that allows to determine in $O\left(\log ^{2} n\right)$ time for each point $q \in P$ the smallest integer $i$ such that $q \in D_{1} \cap \ldots \cap D_{i-1}$ and $q \notin D_{i}$ if such an integer exists. The data structure needs $O(n \log n)$ preprocessing time and space.

Proof: To simplify the presentation we assume $n=2^{k}$. We build a complete binary tree $\mathcal{B}$ with $k$ levels over $n$ leaves with labels $1, \ldots, n$, see Figure 4 . Each inner node $v$ with left child $l$ and right child $r$ is labeled by a set of consecutive integers $\{a(v), \ldots, b(v)\} \subset\{1, \ldots, n\}$ that is recursively defined by $a(v)=a(l)$ and $b(v)=a(r)-1$. For each leaf $w$ we set $a(w)=b(w)=$ label $(w)$. Note that the root is labeled $\{1, \ldots, n / 2\}$. In Figure $4[a, b]$ is shorthand for $\{a, \ldots, b\}$.

A query with a point $q \in P$ consists of following a path from the root to a leaf whose label $i$ is the index of the $q$-furthest point from $p$, in other words $p_{i}=f_{p q}$. In each inner node $v$ the path of a query with point $q$ is determined by testing whether $q \in D_{a(v)} \cap \ldots \cap D_{b(v)}$. If yes the next node of the query path is the right child, otherwise the left child. (Why such a query in deed gives the desired answer can be proven by induction over $k$.)

A query time of $O\left(\log ^{2} n\right)$ can be achieved by storing in each internal node $v$ a decomposition of $D_{a(v)} \cap \ldots \cap D_{b(v)}$ into at most $b(v)-a(v)+2$ vertical strips. The strips are bounded by all verticals through the endpoints of the arcs that for the boundary of $D_{a(v)} \cap \ldots \cap D_{b(v)}$. In this decomposition $q$ can then be located in $O(\log n)$ time, and this has to be done $O(\log n)$ times on the way from the root to a leaf.

In order to construct the tree $\mathcal{B}$ we first build a tree $\mathcal{B}^{\prime}$. The tree $\mathcal{B}^{\prime}$ is also a binary tree over $\{1, \ldots, n\}$, but in $\mathcal{B}^{\prime}$ each internal node $v$ is labeled with the set of the labels of all leaves in the subtree rooted at $v$. The tree $\mathcal{B}^{\prime}$ can be built in a bottom-up fashion since we can construct $D_{1} \cap \ldots \cap D_{2 m}$ from $D_{1} \cap \ldots \cap D_{m}$ and $D_{m+1} \cap \ldots \cap D_{2 m}$ by a merge-sort style procedure in $O(m)$ time. Note that each disk contributes at most one piece to the boundary of the intersection. Hence the construction of $\mathcal{B}^{\prime}$ takes $O(n \log n)$ time (and space) in total.

From $\mathcal{B}^{\prime}$ we obtain $\mathcal{B}$ in three steps. First we make the left child of the root of $\mathcal{B}^{\prime}$ the root of $\mathcal{B}$. Second for each node $v$ we change the pointer from the right child of $v$ to the left child of the sister of $v$, see the dotted arrows in Figure 5. Third, we make each leaf node $w$ with 


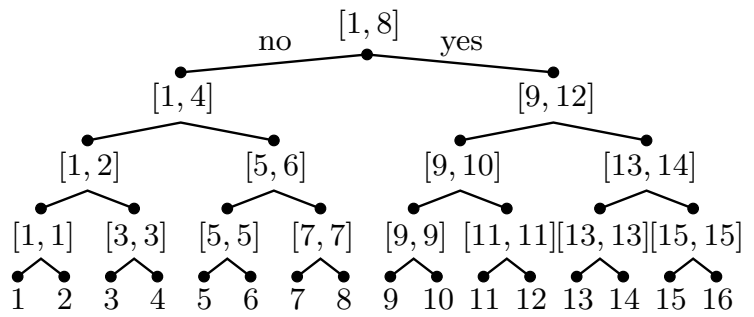

Figure 4: The binary search tree $\mathcal{B}$. Label $[a, b]$ at node $v$ means that the test $q \in D_{a} \cap$ $D_{a+1} \cap \ldots \cap D_{b}$ is performed at $v$ for a query point $q$.

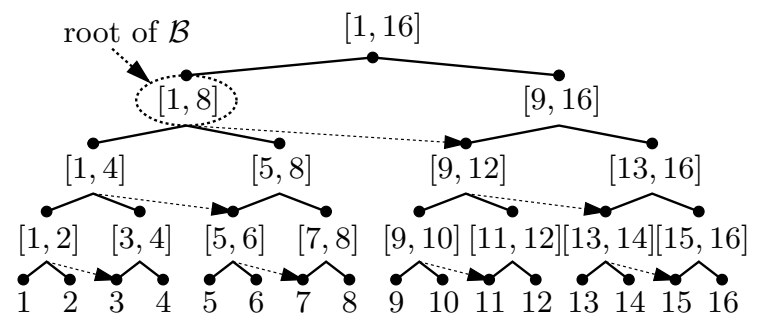

Figure 5: The auxiliary tree $\mathcal{B}^{\prime}$ for the construction of $\mathcal{B}$ (see dotted arrows).

label $i$ in $\mathcal{B}^{\prime}$ an internal node with label $\{i\}$ in $\mathcal{B}$ and add to $w$ a left child with label $i$ and a right child with label $i+1$ as new leaves. The vertical decomposition of each internal node $v$ can then be computed in time linear in the size of the complexity of $D_{a(v)} \cap \ldots \cap D_{b(v)}$. Thus the construction of $\mathcal{B}$ takes $O(n \log n)$ time and space.

The time complexity for querying the data structure can be reduced from $O\left(\log ^{2} n\right)$ to $O(\log n)$ by applying fractional-cascading techniques [CG86]. The time we need to reorganize the tree $\mathcal{B}$ to support fractional cascading is proportional to its space consumption, which is $O(n \log n)$ since there are $O(n)$ items on each level.

Theorem 2.3 There is an algorithm that computes a MSST in $O\left(n^{2} \log n\right)$ time using quadratic space.

Proof: With the procedure described before Lemma 2.2 we can compute, for each $p \in P$, all points $f_{p q}$ that are $q$-farthest from $p$ in $O(n \log n)$ time using the data structure of Lemma 2.2 and fractional cascading. Thus we can compute all points of type $f_{p q}$ in $O\left(n^{2} \log n\right)$ time. Since we can only determine the MSST after computing all points of type $f_{p q}$ we must store them explicitly, which requires quadratic space.

\section{Approximating the minimum-diameter spanning tree}

We first make the trivial observation that the diameter of any monopolar tree on $P$ is at most twice as long as the tree diameter $d_{P}$ of $P$. We use the following notation. Let $\mathcal{T}_{\text {di }}$ be a fixed MDdST and $\mathcal{T}_{\text {mono }}$ a fixed MDmST of $P$. The tree $\mathcal{T}_{\text {di }}$ has minimum diameter among those trees with vertex set $P$ in which all but two nodes - the poles - have degree 1 . The tree $\mathcal{T}_{\text {mono }}$ is a minimum-diameter star with vertex set $P$. Let $x$ and $y$ be the poles of $\mathcal{T}_{\text {di }}$, and let $\delta=|x y|$ be their distance. Finally let $r_{x}\left(r_{y}\right)$ be the length of the longest edge in $\mathcal{T}_{\text {di }}$ incident to $x(y)$ without taking into account the edge $x y$. Thus disks of radius $r_{x}$ and $r_{y}$ centered at $x$ and $y$, respectively, cover $P$. Wlog. we assume $r_{x} \geq r_{y}$.

Ho et al. showed that in the dipolar case (i.e. if there is no monopolar MDST), the disk centered at $y$ cannot be contained by the one centered at $x$. We will need this stability lemma below.

Lemma 3.1 (Stability lemma [HLCW91]) $r_{x}<\delta+r_{y}$. 
In order to get a good approximation of the MDST, we slightly modify the algorithm for the MSST described in Section 2. After computing the $O\left(n^{2}\right)$ points of type $f_{p q}$, we go through all pairs $\{p, q\}$ and consider the tree $\mathcal{T}_{p q}$ with dipole $\{p, q\}$ in which each point is connected to its closer dipole. In Section 2 we were searching for a tree of type $\mathcal{T}_{p q}$ that minimizes $|p q|+\max \left\{\left|f_{p q} p\right|,\left|q f_{q p}\right|\right\}$. Now we go through all trees $\mathcal{T}_{p q}$ to find the tree $\mathcal{T}_{\text {bisect }}$ with minimum diameter, i.e. the tree that minimizes $|p q|+\left|f_{p q} p\right|+\left|q f_{q p}\right|$. Note that the only edge in $\mathcal{T}_{p q}$ that crosses the perpendicular bisector of $p q$ is the edge $p q$ itself. This is of course not necessarily true for the MDdST $\mathcal{T}_{\mathrm{di}}$. We will show the following:

Lemma 3.2 Given a set $P$ of $n$ points in the plane there is a tree with the following two properties: it can be computed in $O\left(n^{2} \log n\right)$ time using $O\left(n^{2}\right)$ storage, and its diameter is at most $4 / 3 \cdot d_{P}$.

Proof: Due to Theorem 2.3 it suffices to show the approximation factor. We will first compute upper bounds for the approximation factors of $\mathcal{T}_{\text {bisect }}$ and $\mathcal{T}_{\text {mono }}$ and then analyze where the minimum of the two takes its maximum.

For the analysis of $\mathcal{T}_{\text {bisect }}$ consider the tree $\mathcal{T}_{x y}$ whose poles are those of $\mathcal{T}_{\text {di }}$. The diameter of $\mathcal{T}_{x y}$ is an upper bound for that of $\mathcal{T}_{\text {bisect }}$. Let $r_{x}^{\prime}\left(r_{y}^{\prime}\right)$ be the length of the longest edge of $\mathcal{T}_{x y}$ incident to $x(y)$ without taking into account the edge $x y$. Note that $r_{x}^{\prime}=\left|x f_{x y}\right|$ and $r_{y}^{\prime}=\left|y f_{y x}\right|$.

Now we compare the diameter of $\mathcal{T}_{x y}$ to that of $\mathcal{T}_{\text {di }}$. Observe that $\max \left\{r_{x}^{\prime}, r_{y}^{\prime}\right\} \leq r_{x}$. This is due to our assumption $r_{x} \geq r_{y}$ and to the fact that $f_{x y}$ and $f_{y x}$ have at most distance $r_{x}$ from both $x$ and $y$. This observation yields diam $\mathcal{T}_{x y}=r_{x}^{\prime}+\delta+r_{y}^{\prime} \leq 2 \max \left\{r_{x}^{\prime}, r_{y}^{\prime}\right\}+\delta \leq 2 r_{x}+\delta$. Now we define two constants $\alpha$ and $\beta$ that only depend on $\mathcal{T}_{\text {di }}$. Let

$$
\alpha=\frac{\delta}{r_{x}+r_{y}} \text { and } \beta=\frac{r_{x}}{r_{y}} \text {. }
$$

Note that $\alpha>0$ and $\beta \geq 1$. Introducing $\alpha$ and $\beta$ yields

$$
\frac{\operatorname{diam} \mathcal{T}_{\text {bisect }}}{\operatorname{diam} \mathcal{T}_{\text {di }}} \leq \frac{\operatorname{diam} \mathcal{T}_{x y}}{\operatorname{diam} \mathcal{T}_{\text {di }}} \leq \frac{2 r_{x}+\delta}{r_{x}+\delta+r_{y}}=\frac{\alpha(1+\beta)+2 \beta}{(1+\alpha)(1+\beta)}=: f_{\text {bisect }}(\alpha, \beta),
$$

since $2 r_{x}=2 \beta\left(r_{x}+r_{y}\right) /(1+\beta)$ and $\delta=\alpha\left(r_{x}+r_{y}\right)$. The function $f_{\text {bisect }}(\alpha, \beta)$ is an upper bound for the approximation factor that $\mathcal{T}_{\text {bisect }}$ achieves.

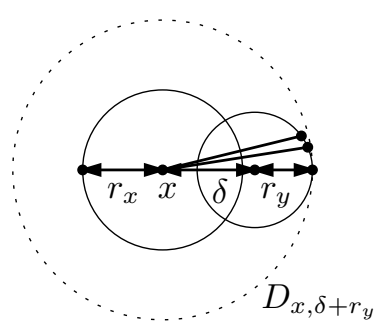

Figure 6: Approximating $\mathcal{T}_{\text {di }}$ with $\mathcal{T}_{\text {mono }}$.

Now we apply our $\alpha$ - $\beta$-analysis to $\mathcal{T}_{\text {mono. }}$. The stability lemma $r_{x}<\delta+r_{y}$ [HLCW91] implies that all points in $P$ are contained in the disk $D_{x, \delta+r_{y}}$ of radius $\delta+r_{y}$ centered at $x$, see Figure 6. Due to that, the diameter of a monopolar tree $\mathcal{T}$ that spans $P$ and is rooted at 
$x$ is at most twice the radius of the disk. We know that diam $\mathcal{T}_{\text {mono }} \leq \operatorname{diam} \mathcal{T}$ since $\mathcal{T}_{\text {mono }}$ is the MDmST of $P$. Thus

$$
\operatorname{diam} \mathcal{T}_{\text {mono }} \leq 2\left(\delta+r_{y}\right)=2 \alpha\left(r_{x}+r_{y}\right)+\frac{2}{1+\beta}\left(r_{x}+r_{y}\right),
$$

since $\delta=\alpha\left(r_{x}+r_{y}\right)$ and $1+\beta=\left(r_{x}+r_{y}\right) / r_{y}$. Using diam $\mathcal{T}_{\mathrm{di}}=(1+\alpha)\left(r_{x}+r_{y}\right)$ yields

$$
\frac{\operatorname{diam} \mathcal{T}_{\text {mono }}}{\operatorname{diam} \mathcal{T}_{\mathrm{di}}} \leq \frac{2 \alpha(1+\beta)+2}{(1+\alpha)(1+\beta)}=: f_{\text {mono }}(\alpha, \beta)
$$

and the function $f_{\text {mono }}(\alpha, \beta)$ is an upper bound of $\mathcal{T}_{\text {mono }}$ 's approximation factor.

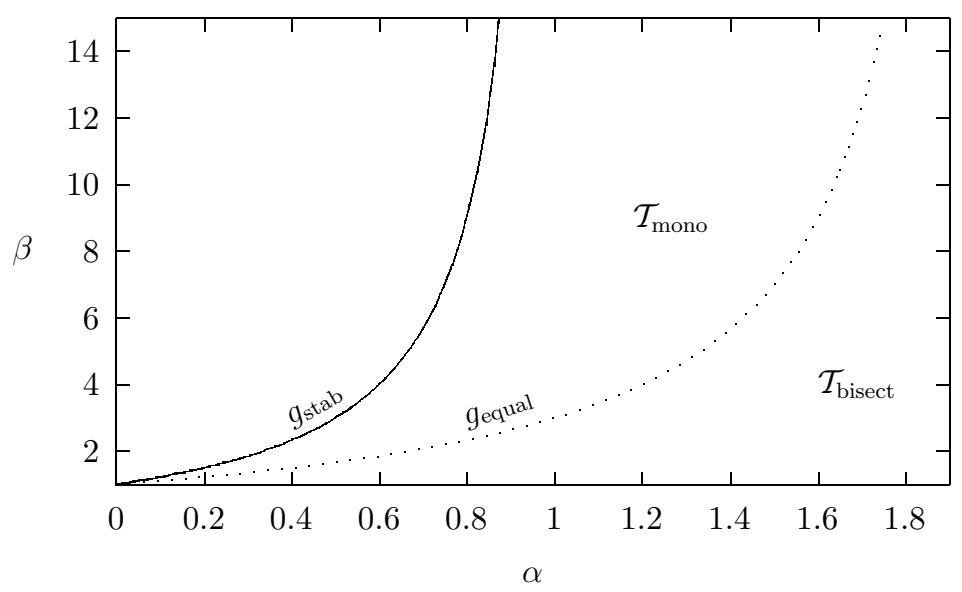

Figure 7: Our bound for $\mathcal{T}_{\text {mono }}$ is better above $g_{\text {equal }}$; that for $\mathcal{T}_{\text {bisect }}$ is better below. To the left of $g_{\text {stab }}$ the tree $\mathcal{T}_{\text {mono }}$ is optimal.

In order to compute the maximum of the minimum of the two bounds we first analyze

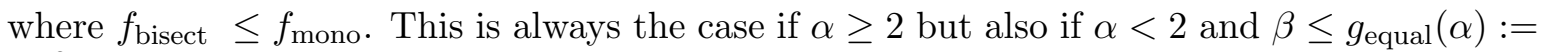
$\frac{\alpha+2}{2-\alpha}$. See Figure 7 for the corresponding regions. Since neither $f_{\text {bisect }}$ nor $f_{\text {mono }}$ have any local or global maxima in the interior of the $(\alpha, \beta)$-range we are interested in, we must consider their boundary values.

1. For $\beta \equiv 1$ the tree $\mathcal{T}_{\text {bisect }}$ is optimal since $f_{\text {bisect }}(\alpha, 1) \equiv 1$.

2. Note that the stability lemma $r_{x} \leq \delta+r_{y}$ is equivalent to $\beta \leq g_{\text {stab }}(\alpha):=\frac{\alpha+1}{1-\alpha}$, see Figure 7 . Along the graph of $g_{\text {stab }}$ the tree $\mathcal{T}_{\text {mono }}$ is optimal since $f_{\text {mono }}\left(\alpha, g_{\text {stab }}(\alpha)\right) \equiv 1$.

3. Along $g_{\text {equal }}$ both functions equal $(3 \alpha+2) /(2 \alpha+2)$. This expression increases monotonically from 1 towards $4 / 3$ when $\alpha$ goes from 0 towards 2 .

The partial derivatives show that $f_{\text {mono }}$ increases while $f_{\text {bisect }}$ decreases monotonically when $\alpha$ goes to infinity. Thus the maximum of $\min \left(f_{\text {mono }}, f_{\text {bisect }}\right)$ is indeed attained at $g_{\text {equal }}$. 


\section{Approximation schemes for the MDST}

In this section we give some fast approximation schemes for the MDST, i.e. factor- $(1+\varepsilon)$ approximation algorithms. The first scheme uses a grid, the second and third the wellseparated pair decomposition, and the forth is a combination of the first and the third method. The reason for this multitude of approaches is that we want to take into account the way the running time depends not only on $n$, the size of the point set, but also on $\varepsilon$, the approximation factor.

Chan [Cha00] uses the following notation. Let $E=1 / \varepsilon$ and let the $O^{*}$-notation be a variant of the $O$-notation that hides terms of type $O\left(\log ^{O(1)} E\right)$. (Such terms come into play e.g. when the use of the floor function is replaced by binary search with precision $\varepsilon$.) Then a linear-time approximation scheme (LTAS) of order $c$ is a scheme with a running time of the form $O^{*}\left(E^{c} n\right)$ for some constant $c$. A strong LTAS of order $c$ has a running time of $O^{*}\left(E^{c}+n\right)$. Our best scheme for approximating the MDST is a strong LTAS of order 5.

\subsection{A grid-based approximation scheme}

The idea of our first scheme is based on a grid which has been used before e.g. to approximate the diameter of a point set [BHP99, Cha00], i.e. the longest distance between any pair of the given points. We lay a grid of $O(E) \times O(E)$ cells over $P$, choose an arbitrary representative point for each cell and use the exact algorithm of Ho et al. [HLCW91] to compute the MDST $\mathcal{T}_{R}$ of the set $R$ of all representative points. By connecting the remaining points in $P \backslash R$ to the pole adjacent to their representatives, we get a dipolar tree $\mathcal{T}_{\varepsilon}$ whose diameter is at most $(1+\varepsilon)$ times the tree diameter $d_{P}$ of $P$.

The details are as follows. Let $M=\max _{p, q \in P}\{|x(p) x(q)|,|y(p) y(q)|\}$ be the edge length of the smallest enclosing square of $P$ and let $l=\varepsilon M /(10 \sqrt{2})$ be the edge length of the square grid cells. Clearly $M \leq d_{P}$. Since each path in $T_{\varepsilon}$ is at most by two edges of length $l \sqrt{2}$ longer than the corresponding path in $T_{R}$ we have diam $T_{\varepsilon} \leq \operatorname{diam} T_{R}+2 l \sqrt{2} \leq \operatorname{diam} T_{R}+\varepsilon d_{P} / 5$. To see that diam $T_{\varepsilon} \leq(1+\varepsilon) d_{P}$ it remains to prove:

Lemma $4.1 \operatorname{diam} \mathcal{T}_{R} \leq(1+4 \varepsilon / 5) d_{P}$.

Proof: Let $\mathcal{T}_{P}$ be a MDST of $P$ that is either mono- or dipolar. Such a tree always exists according to [HLCW91].

Case I: $\mathcal{T}_{P}$ is monopolar. Let $x \in P$ be the pole of $\mathcal{T}_{P}$ and let $\rho_{p} \in R$ be the representative point of $p \in P$. Due to the definition of $T_{R}$ we have

$$
\operatorname{diam} \mathcal{T}_{R} \leq \min _{x^{\prime} \in R} \max _{s \neq t \in R}\left|s x^{\prime}\right|+\left|x^{\prime} t\right| \leq \max _{s \neq t \in R}\left|s \rho_{x}\right|+\left|\rho_{x} t\right| .
$$

(The first two terms are equal if there is a monopolar MDST of $R$, the last two terms are equal if there is a MDmST of $R$ with pole $\rho_{x}$.) By triangle inequality

$$
\operatorname{diam} \mathcal{T}_{R} \leq \max _{s \neq t \in R}|s x|+\left|x \rho_{x}\right|+\left|\rho_{x} x\right|+|x t|,
$$

i.e. we maximize the length of the polygonal chain $\left(s, x, \rho_{x}, x, t\right)$ over all $s \neq t \in R$. By appending edges to points $a$ and $b \in P$ in the grid cells of $s$ and $t$, respectively, the length of the longest chain does not decrease, even if we now maximize over all $a, b \in P$ with $a \neq b$.

$$
\operatorname{diam} \mathcal{T}_{R} \leq \max _{a \neq b \in P}\left|a \rho_{a}\right|+\left|\rho_{a} x\right|+2\left|x \rho_{x}\right|+\left|x \rho_{b}\right|+\left|\rho_{b} b\right| .
$$


Using $\left|a \rho_{a}\right|,\left|x \rho_{x}\right|,\left|\rho_{b} b\right| \leq l \sqrt{2}$ and the triangle inequalities $\left|\rho_{a} x\right| \leq\left|\rho_{a} a\right|+|a x|$ and $\left|x \rho_{b}\right| \leq$ $|x b|+\left|b \rho_{b}\right|$ yields diam $\mathcal{T}_{R} \leq 6 l \sqrt{2}+\max _{a \neq b \in P}|a x|+|x b|=(1+3 \varepsilon / 5) d_{P}$.

Case II: $\mathcal{T}_{P}$ is dipolar. The analysis is very similar to case I, except the chains consist of more pieces. This yields $\operatorname{diam} \mathcal{T}_{R} \leq 8 l \sqrt{2}+\operatorname{diam} \mathcal{T}_{P}=(1+4 \varepsilon / 5) d_{P}$.

Theorem 4.2 A spanning tree $\mathcal{T}_{P}$ of $P$ with diam $\mathcal{T}_{P} \leq(1+1 / E) \cdot d_{P}$ can be computed in $O^{*}\left(E^{6-1 / 3}+n\right)$ time using $O^{*}\left(E^{2}+n\right)$ space.

Proof: In order to determine the grid cell of each point in $P$ without the floor function, we do binary search - once on an $x$ - and once on a $y$-interval of size $M$ until we have reached a precision of $l$, i.e. we need $O(\log E)$ steps for each point. Using Chan's algorithm [Cha02] to compute $T_{R}$ takes $\tilde{O}\left(|R|^{3-1 / 6}\right)$ time and $\tilde{O}(|R|)$ space, where $|R|=O\left(E^{2}\right)$.

\subsection{The well-separated pair decomposition}

Our second scheme uses the well-separated pair decomposition of Callahan and Kosaraju [CK95]. We briefly review this decomposition below.

Definition 4.3 Let $\tau>0$ be a real number, and let $A$ and $B$ be two finite sets of points in $\mathbb{R}^{d}$. We say that $A$ and $B$ are well-separated w.r.t. $\tau$, if there are two disjoint d-dimensional balls $C_{A}$ and $C_{B}$ both of radius $r$ such that $A \subset C_{A}, B \subset C_{B}$, and the distance between $C_{A}$ and $C_{B}$ is at least equal to $\tau r$.

The parameter $\tau$ will be referred to as the separation constant. The following lemma follows easily from Definition 4.3.

Lemma 4.4 Let $A$ and $B$ be two finite sets of points that are well-separated w.r.t. $\tau$, let $x$ and $p$ be points of $A$, and let $y$ and $q$ be points of $B$. Then (i) $|x y| \leq(1+2 / \tau) \cdot|x q|$, (ii) $|x y| \leq(1+4 / \tau) \cdot|p q|$, (iii) $|p x| \leq(2 / \tau) \cdot|p q|$, and (iv) the angle between the line segments $p q$ and py is at most $\arcsin (2 / \tau)$.

Definition 4.5 Let $P$ be a set of $n$ points in $\mathbb{R}^{d}$, and $\tau>0$ a real number. $A$ well-separated pair decomposition (WSPD) for $P$ (w.r.t. $\tau$ ) is a sequence of pairs of non-empty subsets of $P,\left(A_{1}, B_{1}\right),\left(A_{2}, B_{2}\right), \ldots,\left(A_{\ell}, B_{\ell}\right)$, such that

1. $A_{i}$ and $B_{i}$ are well-separated w.r.t. $\tau$ for $i=1,2, \ldots, \ell$, and

2. for any two distinct points $p$ and $q$ of $P$, there is exactly one pair $\left(A_{i}, B_{i}\right)$ in the sequence such that (i) $p \in A_{i}$ and $q \in B_{i}$, or (ii) $q \in A_{i}$ and $p \in B_{i}$,

The integer $\ell$ is called the size of the WSPD. Callahan and Kosaraju show that a WSPD of size $\ell=O\left(\tau^{2} n\right)$ can be computed using $O\left(n \log n+\tau^{2} n\right)$ time and space. 


\subsection{A straight-forward approximation scheme}

The approximation algorithm consists of two subalgorithms: the first algorithm computes a MDmST and the second computes an approximation of the MDdST. We always output the one with smaller diameter. According to [HLCW91] there exists a MDST that is either a monopolar or a dipolar tree. The MDmST can be computed in time $O(n \log n)$, hence we will focus on the problem of computing a MDdST. Let $d_{\text {min }}$ be the diameter of a MDdST and let $\mathcal{S}_{p q}$ denote a spanning tree with dipole $\{p, q\}$ whose diameter is minimum among all such trees. For any dipolar spanning tree $\mathcal{T}$ with dipole $\{u, v\}$ let $r_{u}(\mathcal{T})\left(r_{v}(\mathcal{T})\right)$ be the length of the longest edge of $\mathcal{T}$ incident to $u(v)$ without taking into account the edge $u v$. When it is clear which tree $\mathcal{T}$ we refer to, we will use $r_{u}$ and $r_{v}$.

Observation 4.6 Let $\left(A_{1}, B_{1}\right), \ldots,\left(A_{\ell}, B_{\ell}\right)$ be a WSPD of $P$ w.r.t. $\tau$, and let $p$ and $q$ be any two points in $P$. Then there is a pair $\left(A_{i}, B_{i}\right)$ such that for every point $u \in A_{i}$ and every point $v \in B_{i}$ the inequality diam $\mathcal{S}_{u v} \leq(1+8 / \tau) \cdot \operatorname{diam} \mathcal{S}_{p q}$ holds.

Proof: According to Definition 4.5 there is a pair $\left(A_{i}, B_{i}\right)$ in the WSPD such that $p \in A_{i}$ and $q \in B_{i}$. If $u$ is any point in $A_{i}$ and $v$ is any point in $B_{i}$, then let $\mathcal{T}$ be the tree with poles $u$ and $v$ where $u$ is connected to $v, p$ and each neighbor of $p$ in $\mathcal{S}_{p q}$ except $q$ is connected to $u$, and $q$ and each neighbor of $q$ in $\mathcal{S}_{p q}$ except $p$ is connected to $v$. By Lemma 4.4(ii) $|u v| \leq(1+4 / \tau)|p q|$ and by Lemma 4.4(iii) $r_{u} \leq|u p|+r_{p} \leq 2|p q| / \tau+r_{p}$. Since $\operatorname{diam} \mathcal{T}=r_{u}+|u v|+r_{v}$ we have

$$
\operatorname{diam} \mathcal{T} \leq\left(r_{p}+2 \frac{|p q|}{\tau}\right)+\left(|p q|+4 \frac{|p q|}{\tau}\right)+\left(r_{q}+2 \frac{|p q|}{\tau}\right)<\left(1+\frac{8}{\tau}\right) \cdot \operatorname{diam} \mathcal{S}_{p q} .
$$

The lemma follows due to the minimality of $\mathcal{S}_{u v}$.

A first algorithm is now obvious. For each of the $O\left(\tau^{2} n\right)$ pairs $\left(A_{i}, B_{i}\right)$ in a WSPD of $P$ w.r.t. $\tau=8 E$ pick any point $p \in A_{i}$ and any point $q \in B_{i}$, sort $P$ according to distance from $p$, and compute $\mathcal{S}_{p q}$ in linear time by checking every possible radius of a disk centered at $p$ as in [HLCW91].

Lemma $4.7 A$ dipolar tree $\mathcal{T}$ with diam $\mathcal{T} \leq(1+1 / E) \cdot d_{\min }$ can be computed in $O\left(E^{2} n^{2} \log n\right)$ time using $O\left(E^{2} n+n \log n\right)$ space.

\subsection{A fast approximation scheme}

Now we describe a more involved algorithm. It is faster than the previous algorithm for $n=\Omega(E)$. We will prove its correctness in Section 4.5.

Theorem 4.8 A dipolar tree $\mathcal{T}$ with diam $\mathcal{T} \leq(1+1 / E) \cdot d_{\min }$ can be computed in $O\left(E^{3} n+\right.$ En $\log n)$ time using $O\left(E^{2} n+n \log n\right)$ space.

The idea of the algorithm is again to check a linear number of pairs of points, using the WSPD, but to speed up the computation of the disks around the two poles. Note that we need to find a close approximation of the diameters of the disks to be able to guarantee a $(1+\varepsilon)$-approximation of the MDdST. Obviously we cannot afford to try all possible disks for all possible pairs of poles. Instead of checking the disks we will show in the analysis that it suffices to check a constant number of partitions of the points among the poles. The partition 
of points is done by cuts that are orthogonal to the line through the poles. We cannot afford to do this for each possible pair. Instead we select a constant number of orientations and use a constant number of orthogonal cuts for each orientation. For each cut we calculate for each point in $P$ the approximate distance to the farthest point on each side of the cut. Below we give a more detailed description of the algorithm. For its pseudocode refer to Algorithm 1.

Phase 1: Initializing. Choose an auxiliary positive constant $\kappa<\min \{0.9 \varepsilon, 1 / 2\}$. As will be clear later, this parameter can be used to fine-tune which part of the algorithm contributes how much to the uncertainty and to the running time. In phase 3 the choice of the separation constant $\tau$ will depend on the value of $\kappa$ and $\varepsilon$.

Definition 4.9 A set of points $P$ is said to be l-ordered if the points are ordered with respect to their orthogonal projection onto the line $l$.

Let $l_{i}$ be the line with angle $i \pi / \gamma$ to the horizontal line, where $\gamma=\lceil 4 / \kappa\rceil$. This implies that for an arbitrary line $l$ there exists a line $l_{i}$ such that $\angle l_{i} l \leq \pi /(2 \gamma)$. For $i=1, \ldots, \gamma$, let $F_{i}$ be a list of the input points sorted according to the $l_{i}$-ordering. The time to construct these lists is $O(\gamma n \log n)$.

For each $l_{i}$, rotate $P$ and $l_{i}$ such that $l_{i}$ is horizontal. For simplicity we denote the points in $P$ from left to right on $l_{i}$ by $p_{1}, \ldots, p_{n}$. Let $d_{i}$ denote the horizontal distance between $p_{1}$ and $p_{n}$. Let $b_{i j}, 1 \leq j \leq \gamma$, be a marker on $l_{i}$ at distance $j d_{i} /(\gamma+1)$ to the right of $p_{1}$. Let $L_{i j}$ and $R_{i j}$ be the set of points in $P$ to the left and to the right of the vertical $\beta_{i j}$ through $b_{i j}$, respectively.

For each marker $b_{i j}$ on $l_{i}$ we construct $\gamma$ pairs of lists, denoted $L_{i j k}^{\prime}$ and $R_{i j k}^{\prime}$, where $1 \leq k \leq \gamma$. The list $L_{i j k}^{\prime}\left(R_{i j k}^{\prime}\right)$ contains the points in $L_{i j}\left(R_{i j}\right)$ sorted according to the $l_{k}$-ordering. Such a list can be constructed in $O(n)$ time since the ordering is given by $F_{k}$ : we just have to filter out the points in $F_{k}$ that are on the "wrong" side of $\beta_{i j}$. (Actually it is not necessary to store the whole lists $L_{i j k}^{\prime}$ and $R_{i j k}^{\prime}$ : we only need to store the first and the last point in each list.) Hence the total time complexity needed to construct the lists is $O\left(\gamma^{3} n+\gamma n \log n\right)$, see lines 1-17 in Algorithm 1. These lists will help us to compute an approximate farthest neighbor in $L_{i j}$ and $R_{i j}$ for each point $p \in P$ in time $O(\gamma)$, as we describe below.

Phase 2: Computing approximate farthest neighbors. Let the approximate distance of a point $q$ from $p$ be the maximum distance among all projections of $q$ onto the lines $l_{k}$. Now let the approximate farthest neighbor $N(p, i, j, L)$ of $p$ be the point $q \in L_{i j}$ with maximum approximate distance from $p$. Each $N(p, i, j, L)$ can be computed in time $O(\gamma)$ by taking the farthest point from $p$ over all first and last elements of $L_{i j k}^{\prime}$ with $k=1, \ldots, \gamma$. Define and compute $N(p, i, j, R)$ analogously. Hence the total time complexity of phase 2 is $O\left(\gamma^{3} n\right)$, as there are $O\left(\gamma^{2} n\right)$ triples of type $(p, i, j)$. The error we make by using approximate farthest neighbors is small:

Observation 4.10 If $p$ is any point in $P, p_{L}$ the point in $L_{i j}$ farthest from $p$ and $p_{R}$ the point in $R_{i j}$ farthest from $p$, then

(a) $\left|p p_{L}\right| \leq(1+\kappa / 24) \cdot|p N(p, i, j, L)|$ and

(b) $\left|p p_{R}\right| \leq(1+\kappa / 24) \cdot|p N(p, i, j, R)|$. 


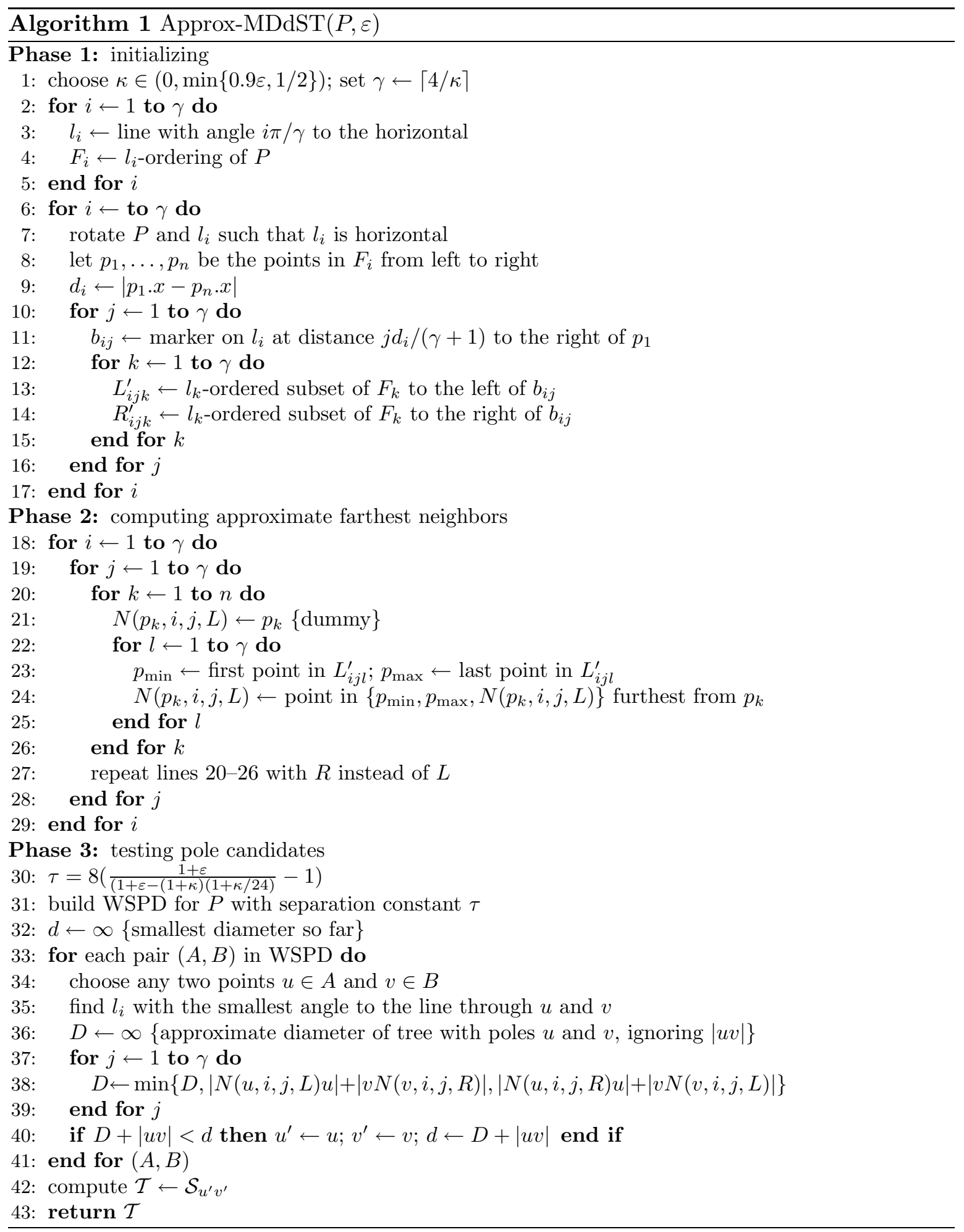


Proof: Due to symmetry it suffices to check (a). If the algorithm did not select $p_{L}$ as farthest neighbor it holds that for each of the $l_{k}$-orderings there is a point farther from $p$ than $p_{L}$. Hence $p_{L}$ must lie within a symmetric $2 \gamma$-gon whose edges are at distance $|p N(p, i, j, L)|$ from $p$. This implies that $\left|p p_{L}\right| \leq|p N(p, i, j, L)| / \cos (\pi /(2 \gamma)) \leq|p N(p, i, j, L)| / \cos (\pi \kappa / 8)$ using $\gamma=\lceil 4 / \kappa\rceil$. Thus it remains to show that $1 / \cos (\pi \kappa / 8) \leq 1+\kappa / 24$. Since $\cos x \geq 1-x^{2} / 2$ for any $x$, the claim is true if $1-\pi^{2} \kappa^{2} / 128 \geq 1 /(1+\kappa / 24)$. This inequality holds for all $0<\kappa \leq 1 / 2$.

Phase 3: Testing pole candidates. Compute the WSPD for $P$ with separation constant $\tau$. To be able to guarantee a $(1+\varepsilon)$-approximation algorithm the value of $\tau$ will depend on $\varepsilon$ and $\kappa$ as follows:

$$
\tau=8\left(\frac{1+\varepsilon}{1+\varepsilon-(1+\kappa)(1+\kappa / 24)}-1\right) .
$$

Note that the above formula implies that there is a trade-off between the values $\tau$ and $\kappa$, which can be used to fine-tune which part of the algorithm contributes how much to the uncertainty and to the running time. Setting for instance $\kappa$ to $0.9 \varepsilon$ yields for $\varepsilon$ small $16 / \varepsilon+$ $15<\tau / 8<32 / \varepsilon+31$, i.e. $\tau=\Theta(1 / \varepsilon)$. For each pair $(A, B)$ in the decomposition we select two arbitrary points $u \in A$ and $v \in B$. Let $l_{(u, v)}$ be the line through $u$ and $v$. Find the line $l_{i}$ that minimizes the angle between $l_{i}$ and $l_{(u, v)}$. That is, the line $l_{i}$ is a close approximation of the direction of the line through $u$ and $v$. From above we have that $l_{i}$ is divided into $\gamma+1$ intervals of length $d_{i} /(\gamma+1)$. For each $j, 1 \leq j \leq \gamma$, compute $\min (|N(u, i, j, L) u|+|v N(v, i, j, R)|,|N(u, i, j, R) u|+|v N(v, i, j, L)|)$. The smallest of these $O(\gamma)$ values is saved, and is a close approximation of diam $\mathcal{S}_{u v}-|u v|$, which will be shown below.

The number of pairs in the WSPD is $O\left(\tau^{2} n\right)$, which implies that the total running time of the central loop of this phase (lines 33-41 in Algorithm 1) is $O\left(\gamma \cdot \tau^{2} n\right)$. Building the WSPD and computing $\mathcal{S}_{u^{\prime} v^{\prime}}$ takes an extra $O\left(\tau^{2} n+n \log n\right)$ time. Thus the whole algorithm runs in $O\left(\gamma^{3} n+\gamma \tau^{2} n+\gamma n \log n\right)$ time and uses $O\left(n \log n+\gamma^{2} n+\tau^{2} n\right)$ space. Setting $\kappa=0.9 \varepsilon$ yields $\gamma=O(E)$ and $\tau=O(E)$ and thus the time and space complexities we claimed.

\subsection{The proof of correctness for Theorem 4.8}

It remains to prove that the diameter of the dipolar tree that we compute is indeed at most $(1+\varepsilon) d_{\min }$.

From Observation 4.6 we know that we will test a pair of poles $u$ and $v$ for which diam $\mathcal{S}_{u v} \leq(1+8 / \tau) d_{\min }=\frac{1+\varepsilon}{(1+\kappa)(1+\kappa / 24)} d_{\min }$. The equality actually explains our choice of $\tau$. In this section we will prove that our algorithm always computes a dipolar tree whose diameter is at most $(1+\kappa)(1+\kappa / 24)$ diam $\mathcal{S}_{u v}$ and thus at most $(1+\varepsilon) d_{\min }$.

Consider the tree $\mathcal{S}_{u v}$. For simplicity we rotate $P$ such that the line $l$ through $u$ and $v$ is horizontal and $u$ lies to the left of $v$, as illustrated in Figure 8a. Let $\delta=|u v|$. Our aim is to prove that there exists an orthogonal cut that splits the point set $P$ into two sets such that the tree obtained by connecting $u$ to all points to the left of the cut and connecting $v$ to all points to the right of the cut will give a tree whose diameter is a $(1+\kappa)$-approximation of diam $\mathcal{S}_{u v}$. Since the error introduced by approximating the farthest neighbor distances is not more than a factor of $(1+\kappa / 24)$ according to Observation 4.10 , this will prove the claim in the previous paragraph. 

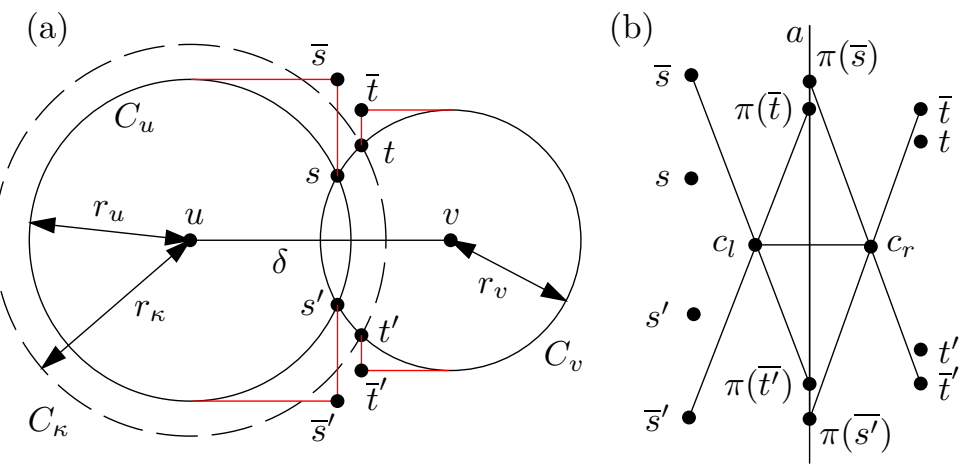

Figure 8: A valid cut.

Denote by $C_{u}$ and $C_{\kappa}$ the circles with center at $u$ and with radius $r_{u}$ and $r_{\kappa}=r_{u}+\kappa z$ respectively, where $z=\operatorname{diam} \mathcal{S}_{u v}=\delta+r_{u}+r_{v}$. Denote by $C_{v}$ the circle with center at $v$ and with radius $r_{v}$. Let $s$ and $s^{\prime}\left(t\right.$ and $t^{\prime}$ ) be two points on $C_{u}\left(C_{v}\right)$ such that if $C_{u}\left(C_{\kappa}\right)$ and $C_{v}$ intersect then $s$ and $s^{\prime}\left(t\right.$ and $\left.t^{\prime}\right)$ are the two intersection points, where $s(t)$ lies above $s^{\prime}\left(t^{\prime}\right)$. Otherwise, if $C_{u}\left(C_{\kappa}\right)$ and $C_{v}$ do not intersect, then $s=s^{\prime}\left(t=t^{\prime}\right)$ is the intersection of the line segment $(u, v)$ and $C_{u}\left(C_{v}\right)$, see Figure 8a.

We say that a cut with a line $l_{\kappa}$ is valid iff all points in $P$ to the left of $l_{\kappa}$ are contained in $C_{\kappa}$ and all points of $P$ to the right of $l_{\kappa}$ are contained in $C_{v}$. A valid cut guarantees a dipolar tree whose diameter is at most $\delta+r_{\kappa}+r_{v}=(1+\kappa) \cdot \operatorname{diam} \mathcal{S}_{u v}$.

We will prove that the algorithm above always considers a valid cut. For simplicity we assume that $r_{u}\left(\mathcal{S}_{u v}\right) \geq r_{v}\left(\mathcal{S}_{u v}\right)$. We will show that there always exists a marker $b_{i j}$ on $l_{i}$ such that cutting $l_{i}$ orthogonally through $b_{i j}$ is valid. Actually it is enough to show that the two requirements below are valid for any $\mathcal{S}_{u v}$. For a point $p$, denote the $x$-coordinate and the $y$-coordinate of $p$ by $p . x$ and $p . y$, respectively. For simplicity we set $u=(0,0)$.

$$
\begin{aligned}
& \text { (i) } \frac{z}{\gamma+1} \cdot \frac{1}{\cos \frac{\pi}{2 \gamma}} \leq \frac{1}{2}(t . x-s . x), \text { and } \\
& \text { (ii) } \tan \frac{\pi}{2 \gamma} \leq \frac{t . x-s . x}{2\left(r_{u}\left(\mathcal{S}_{u v}\right)+r_{v}\left(\mathcal{S}_{u v}\right)\right)}
\end{aligned}
$$

The reason for this will now be explained. First we need to define some additional points. The reader is encouraged to study Figure 8 for a visual description. Let $\bar{s}=\left(s . x, r_{u}\right)$, $\bar{s}^{\prime}=\left(s^{\prime} \cdot x,-r_{u}\right), \bar{t}=\left(t . x, r_{v}\right)$ and $\bar{t}^{\prime}=\left(t^{\prime} \cdot x,-r_{v}\right)$. Let $a$ be the perpendicular bisector of the projections of $s$ and $t$ on the $x$-axis and let $\pi$ be the orthogonal projection of the plane on $a$. Now we can define $c_{l}$ to be the intersection point of the lines $\left(\bar{s}, \pi\left(\bar{t}^{\prime}\right)\right)$ and $\left(\bar{s}^{\prime}, \pi(\bar{t})\right)$, and $c_{r}$ to be the intersection point of the lines $\left(\bar{t}, \pi\left(\bar{s}^{\prime}\right)\right)$ and $\left(\bar{t}^{\prime}, \pi(\bar{s})\right)$.

It now follows that any bisector $l^{\prime}$ that intersects the three line segments $(\bar{s}, \bar{t}),\left(c_{l}, c_{r}\right)$ and $\left(\bar{s}^{\prime}, \bar{t}^{\prime}\right)$, will be a valid cut. This follows since all points to the left of $l^{\prime}$ will be connected to $u$ and all points to the right of $l^{\prime}$ will be connected to $v$, and the diameter of that tree will, obviously, be bounded by $\delta+\left(r_{u}\left(\mathcal{S}_{u v}\right)+\kappa z\right)+r_{u}\left(\mathcal{S}_{u v}\right)$ which is a $(1+\kappa)$-approximation of $\operatorname{diam} \mathcal{S}_{u v}$.

From the algorithm we know that (a) there is a line $l_{i}$ such that $\angle\left(l_{i}, l_{(u, v)}\right) \leq \pi /(2 \gamma)$, and that (b) there are $\gamma$ orthogonal cuts of $l_{i}$ that define equally many partitions of $P$. The 
distance between two adjacent orthogonal cuts of $l_{i}$ is at most $z /(\gamma+1)$. This implies that the length of the largest interval on $l_{(u, v)}$ that is not intersected by any of these orthogonal cuts is at most

$$
\frac{1}{\cos \frac{\pi}{2 \gamma}} \cdot \frac{z}{\gamma+1}
$$

Hence requirement (i) ensures that for every $\mathcal{S}_{u v}$ the distance $\left|c_{l} c_{r}\right|=(t . x-s . x) / 2$ must be large enough to guarantee that there is an orthogonal cut of $l_{i}$ that intersects it.

An orthogonal cut of $l_{i}$ has an angle of at least $\pi / 2-\pi /(2 \gamma)$ to $l_{(u, v)}$. To ensure that an orthogonal cut of $l_{i}$ that intersects the line segment $\overline{c_{l} c_{r}}$ also passes between $\bar{s}$ and $\bar{t}$ and between $\bar{s}^{\prime}$ and $\bar{t}^{\prime}$ it suffices to add requirement (ii).

It remains to prove the following lemma which implies that for every $\mathcal{S}_{u v}$ there is a valid orthogonal cut.

Lemma 4.11 For any $u, v \in P(u \neq v)$ the tree $\mathcal{S}_{u v}$ fulfills requirements (i) and (ii).

Proof: The tree $\mathcal{S}_{u v}$ can be characterized by the relationship of the two ratios

$$
\alpha:=\frac{\delta}{r_{u}+r_{v}} \quad \text { and } \quad F:=\frac{1+\kappa / 2}{1-\kappa / 2} .
$$

We distinguish three cases: (1) $\alpha<1$, (2) $1 \leq \alpha \leq F$, and (3) $\alpha>F$. For each of these three cases we will show that $\mathcal{S}_{u v}$ fulfills the two requirements.

Case 1: Using the following two straight-forward equalities, $s . x^{2}+s \cdot y^{2}=r_{u}^{2}$ and $(\delta-s . x)^{2}+$ $s . y^{2}=r_{v}^{2}$, we obtain that $s . x=\left(\delta^{2}+r_{u}^{2}-r_{v}^{2}\right) /(2 \delta)$. A similar calculation for $t . x$ yields $t . x=\left(\delta^{2}+r_{\kappa}^{2}-r_{v}^{2}\right) /(2 \delta)$. Inserting these values gives $t . x-s . x=\left(\kappa^{2} z^{2}+2 \kappa z r_{u}\right) /(2 \delta)$. The fact that $\alpha \leq F$ allows us to further simplify the expression for $t . x-s . x$ by using the following two expressions:

$$
\frac{z}{\delta}=\frac{\delta+r_{u}+r_{v}}{\delta}=1+\frac{r_{u}+r_{v}}{\delta} \geq \frac{2}{1+\kappa / 2}, \quad \text { and } \quad \frac{r_{u}}{\delta} \geq \frac{1-\kappa / 2}{2(1+\kappa / 2)} .
$$

From this we obtain that

$$
t . x-s . x=\frac{\kappa z}{2}\left(\frac{\kappa z}{\delta}+\frac{2 r_{u}}{\delta}\right)>\frac{\kappa z}{2} .
$$

This fulfills requirement (i) since

$$
\frac{z}{\gamma+1} \cdot \frac{1}{\cos \frac{\pi}{2 \gamma}} \leq \frac{\kappa z}{4} \leq \frac{1}{2}(t . x-s . x) .
$$

For requirement (ii) note that $\tan \pi /(2 \gamma) \leq 2 \kappa \tan \pi / 16<2 \kappa / 5$. Since $\kappa \leq 1 / 2$ we get that $z / \delta \geq 2 /(1+\kappa / 2) \geq 8 / 5$. Combining this inequality, Equality 1 , and our assumption that $r_{u} \geq r_{v}$ shows that requirement (ii) is also fulfilled:

$$
\frac{t . x-s . x}{2\left(r_{u}+r_{v}\right)} \geq \frac{\kappa z}{4 \delta}\left(\frac{2 r_{u}+\kappa z}{r_{u}+r_{v}}\right) \geq \frac{\kappa z}{4 \delta} \geq \frac{2 \kappa}{5} .
$$

Case 2: In this case we argue in the same manner as in the previous case. Using the fact that $s . x=r_{u}$ and $t . x=\left(\delta^{2}+r_{\kappa}^{2}-r_{v}^{2}\right) /(2 \delta)$ yields

$$
t . x-s . x \geq \frac{\kappa z}{2}\left(\frac{\kappa z}{\delta}+\frac{2 r_{u}}{\delta}\right)>\frac{\kappa z}{2} .
$$


The rest of the proof is exactly as in case 1.

Case 3: The first requirement is already shown to be fulfilled since $t . x-s . x \geq \delta-r_{u}-r_{v} \geq$ $\kappa z / 2$, hence it remains to show requirement (ii). We have

$$
\frac{t . x-s . x}{2\left(r_{u}+r_{v}\right)} \geq \frac{\delta-\left(r_{u}+r_{v}\right)}{2\left(r_{u}+r_{v}\right)}
$$

plugging in the values gives $\kappa /(2-\kappa)$, which is at least $2 \kappa / 5$. The lemma follows.

The lemma says that for every dipole $\{u, v\}$ there exists a line $a$ such that the dipolar tree obtained by connecting all the points on one side of $a$ to $u$ and all the points on the opposite side to $v$, is a $(1+\kappa)$-approximation of $\mathcal{S}_{u v}$.

\subsection{Putting things together}

Combining grid- and WSPD-based approach yields a strong LTAS of order 5:

Theorem 4.12 A spanning tree $\mathcal{T}$ of $P$ with diam $\mathcal{T} \leq(1+1 / E) d_{P}$ can be computed in $O^{*}\left(E^{5}+n\right)$ time using $O\left(E^{4}+n\right)$ space.

Proof: Applying Algorithm 1 to the set $R \subseteq P$ of the $O\left(E^{2}\right)$ representative points takes $O\left(E^{3}|R|+E|R| \log |R|\right)$ time using $O\left(E^{2}|R|+|R| \log |R|\right)$ space according to Theorem 4.8. Connecting the points in $P \backslash R$ to the poles adjacent to their representative points yields a $(1+\varepsilon)$-approximation of the MDdST of $P$ within the claimed time and space bounds as in Section 4.1. The difference is that now the grid cells must be slightly smaller in order to compensate for the fact that we now approximate the MDdST of $R$ rather than compute it exactly. A $(1+\varepsilon)$-approximation of the MDmST of $P$ can be computed via the grid and an exact algorithm of Ho et al. [HLCW91] in $O^{*}\left(E^{2}+n\right)$ time using $O\left(E^{2}+n\right)$ space. Of the two trees the one with smaller diameter is a $(1+\varepsilon)$-approximation of the MDST of $P$.

\section{Conclusions}

On the one hand we have presented a new planar facility location problem, the discrete minimum-sum two-center problem that mediates between the discrete two-center problem and the minimum-diameter dipolar spanning tree. We have shown that there is an algorithm that computes the corresponding MSST in $O\left(n^{2} \log n\right)$ time and that a variant of this tree is a factor- $4 / 3$ approximation of the MDST. Is there a near quadratic-time algorithm for the MSST that uses $o\left(n^{2}\right)$ space?

On the other hand we have given a number of fast approximation schemes for the MDST. The fastest is a combination of a grid-based approach with an algorithm that uses the wellseparated pair decomposition. It computes in $O^{*}\left(\varepsilon^{-5}+n\right)$ time a tree whose diameter is at most $(1+\varepsilon)$ times that of a MDST. Such an algorithm is called a strong linear-time approximation scheme of order 5 . Spriggs et al. $\left[\mathrm{SKB}^{+} 02\right]$ recently improved our result by giving a strong LTAS of order 3 whose space consumption is linear in $n$ and does not depend on $\varepsilon$. Is order 3 optimal? Is there an exact algorithm that is faster than Chan's [Cha02]? Is there a non-trivial lower bound on the computation time needed for the exact MDST?

Our scheme also works for higher-dimensional point sets, but the running time increases exponentially with the dimension. Linear-time approximation schemes for the discrete twocenter problem and the MSST can be constructed similarly. 


\section{Acknowledgments}

We thank an anonymous referee of an earlier version of this paper for suggesting Theorem 4.2. We also thank Pankaj K. Agarwal for pointing us to [Cha02] and Timothy Chan for sending us an updated version of [Cha02].

\section{References}

[AM95] Pankaj K. Agarwal and Jiří Matoušek. Dynamic half-space range reporting and its applications. Algorithmica, 13:325-345, 1995.

[ASW98] Pankaj K. Agarwal, Micha Sharir, and Emo Welzl. The discrete 2-center problem. Discrete 83 Computational Geometry, 20, 1998.

[BHP99] Gill Barequet and Sariel Har-Peled. Efficiently approximating the minimumvolume bounding box of a point set in three dimensions. In Proc. 10th Annual ACM-SIAM Symposium on Discrete Algorithms (SODA'99), pages 82-91, Baltimore MA, 17-19 January 1999.

[CG86] Bernard Chazelle and Leonidas J. Guibas. Fractional cascading: I. A data structuring technique. Algorithmica, 1(3):133-162, 1986.

[Cha00] Timothy M. Chan. Approximating the diameter, width, smallest enclosing cylinder, and minimum-width annulus. In Proc. 16th Annual Symposium on Computational Geometry (SoCG'00), pages 300-309, New York, 12-14 June 2000. ACM Press.

[Cha02] Timothy M. Chan. Semi-online maintenance of geometric optima and measures. In Proc. 13th ACM-SIAM Symposium on Discrete Algorithms (SODA'02), pages 474-483, San Francisco, 6-8 January 2002.

[CK95] Paul B. Callahan and S. Rao Kosaraju. A decomposition of multidimensional point sets with applications to $k$-nearest-neighbors and $n$-body potential fields. Journal of the ACM, 42(1):67-90, January 1995.

[GJ79] Michael R. Garey and David S. Johnson. Computers and Intractability: A Guide to the Theory of NP-Completeness. W. H. Freeman, New York, NY, 1979.

[HLCW91] Jan-Ming Ho, D. T. Lee, Chia-Hsiang Chang, and C. K. Wong. Minimum diameter spanning trees and related problems. SIAM Journal on Computing, 20(5):987997, October 1991.

[HT95] Refael Hassin and Arie Tamir. On the minimum diameter spanning tree problem. Information Processing Letters, 53(2):109-111, 1995.

$\left[\mathrm{SKB}^{+} 02\right] \quad$ M. J. Spriggs, J. M. Keil, S. Bespamyatnikh, M. Segal, and J. Snoeyink. Computing a $(1+\epsilon)$-approximate geometric minimum-diameter spanning tree. Private communication, 2002. 